\title{
The generalized numerical range of a set of matrices
}

\author{
Pan-Shun Lau, Chi-Kwong Li, Yiu-Tung Poon, and Nung-Sing Sze
}

\begin{abstract}
For a given set of $n \times n$ matrices $\mathcal{F}$, we study the union of the $C$-numerical ranges of the matrices in the set $\mathcal{F}$, denoted by $W_{C}(\mathcal{F})$. We obtain basic algebraic and topological properties of $W_{C}(\mathcal{F})$, and show that there are connections between the geometric properties of $W_{C}(\mathcal{F})$ and the algebraic properties of $C$ and the matrices in $\mathcal{F}$. Furthermore, we consider the starshapedness and convexity of the set $W_{C}(\mathcal{F})$. In particular, we show that if $\mathcal{F}$ is the convex hull of two matrices such that $W_{C}(A)$ and $W_{C}(B)$ are convex, then the set $W_{C}(\mathcal{F})$ is star-shaped. We also investigate the extensions of the results to the joint $C$-numerical range of an $m$-tuple of matrices.
\end{abstract}

AMS classification. 15A60.

Keywords. Numerical range, convex set, star-shaped set.

\section{Introduction}

Let $M_{n}$ be the set of all $n \times n$ complex matrices. The numerical range of $A \in M_{n}$ is defined by

$$
W(A)=\left\{x^{*} A x: x \in \mathbb{C}^{n}, x^{*} x=1\right\},
$$

which is a useful tool for studying matrices and operators; for example, see [8, Chapter 22] and [9, Chapter 1]. In particular, there is an interesting interplay between the geometrical properties of $W(A)$ and the algebraic properties of $A$.

In this paper, for a nonempty set $\mathcal{F}$ of matrices in $M_{n}$, we consider

$$
W(\mathcal{F})=\cup\{W(A): A \in \mathcal{F}\} .
$$

We show that there are also interesting connections between the geometrical properties of $W(\mathcal{F})$ and the properties of the matrices in $\mathcal{F}$.

The study has motivations from different topics. We mention two of them in the following. The first one arises in the study of Crouzeix's conjecture asserting that for any $A \in M_{n}$,

$$
\|f(A)\| \leq 2 \max \{|f(\mu)|: \mu \in W(A)\}
$$

for any complex polynomial $f(z)$, where $\|A\|$ denotes the operator norm of $A$, see [7]. Instead of focusing on a single matrix $A \in M_{n}$, one may consider a complex convex set $K$ and show that

$$
\|f(A)\| \leq 2 \max \{|f(\mu)|: \mu \in K\}
$$


whenever $W(A) \subseteq K$. One readily shows that this is equivalent to the Crouzeix's conjecture. In fact, one may focus on the case when $K$ is a convex polygon (including interior) because $W(A)$ can always be approximated by convex polygons from inside or outside.

Another motivation comes from quantum information science. In quantum mechanics, a pure state in $M_{n}$ is a rank one orthogonal projection of the form $x x^{*}$ for some unit vector $x \in \mathbb{C}^{n}$, and a general state is a density matrix, which is a convex combination of pure states. For a measurement operator $A \in M_{n}$, which is usually Hermitian, the measurement of a state $P$ is computed by $\langle A, P\rangle=\operatorname{tr}(A P)$. As a result,

$$
W(A)=\left\{x^{*} A x: x \in \mathbb{C}^{n}, x^{*} x=1\right\}=\left\{\operatorname{tr}\left(A x x^{*}\right): x \in \mathbb{C}^{n}, x^{*} x=1\right\}
$$

can be viewed as the set of all possible measurements on pure states for a given measurement operator $A$. By the convexity of the numerical range, we have

$$
W(A)=\left\{\operatorname{tr}\left(A x x^{*}\right): x \in \mathbb{C}^{n}, x^{*} x=1\right\}=\{\operatorname{tr}(A P): P \text { is a general state }\} .
$$

So, $W(A)$ actually contains all possible measurements on general states for a given measurement operator $A$. If we consider all possible measurements under a set $\mathcal{F}$ of measurement operators, then it is natural to study $W(\mathcal{F})$. In fact, if we know the set $W(\mathcal{F})$, we may deduce some properties about the measurement operators in $\mathcal{F}$. For example, one can show that

(a) $W(\mathcal{F})=\{\mu\}$ if and only if $\mathcal{F}=\{\mu I\}$.

(b) $W(\mathcal{F}) \subseteq \mathbb{R}$ if and only if all matrices in $\mathcal{F}$ are Hermitian.

(c) $W(\mathcal{F}) \subseteq[0, \infty)$ if and only if all matrices in $\mathcal{F}$ are positive semi-definite.

It is worth pointing out that if $A \in M_{n}$ is not Hermitian, one may consider the Hermitian decomposition $A=A_{1}+i A_{2}$ such that $A_{1}, A_{2}$ are Hermitian, and identify $W(A)$ as the joint numerical range of $\left(A_{1}, A_{2}\right)$ defined by

$$
W\left(A_{1}, A_{2}\right)=\left\{\left(x^{*} A_{1} x, x^{*} A_{2} x\right): x \in \mathbb{C}^{n}, x^{*} x=1\right\} \subseteq \mathbb{R}^{2} .
$$

One can study the joint numerical range of $k$-tuple of Hermitian matrices $\left(A_{1}, \ldots, A_{k}\right)$ defined by

$$
W\left(A_{1}, \ldots, A_{k}\right)=\left\{\left(x^{*} A_{1} x, \ldots, x^{*} A_{k} x\right): x \in \mathbb{C}^{n}, x^{*} x=1\right\} \subseteq \mathbb{R}^{k} .
$$

Accordingly, one may consider the joint numerical range $W(\mathcal{F}) \subseteq \mathbb{R}^{k}$ of a set $\mathcal{F}$ of $k$-tuple of Hermitian matrices in $M_{n}$.

It turns out that we can study $W(\mathcal{F})$ under a more general setting. For a matrix $C \in M_{n}$, the $C$-numerical range of $A \in M_{n}$ is defined by

$$
W_{C}(A)=\left\{\operatorname{tr}\left(C U^{*} A U\right): U \text { is unitary }\right\}
$$

which has been studied by many researchers in connection to different topics; see [10] and its references. In particular, the $C$-numerical range is useful in the study of quantum control 
and quantum information; for example, see [16]. Denote by $\left\{E_{11}, E_{12}, \ldots, E_{n n}\right\}$ the standard basis for $M_{n}$. When $C=E_{11}, W_{C}(A)$ reduces to $W(A)$; if $C$ is a rank $m$ orthogonal projection, then $W_{C}(A)$ reduces to the $m$ th-numerical range of $A$; see [ [8, 9, 12]. For a non-empty set $\mathcal{F}$ of matrices in $M_{n}$, we consider

$$
W_{C}(\mathcal{F})=\cup\left\{W_{C}(A): A \in \mathcal{F}\right\}
$$

In Section 2, we study the connection between the geometric properties of $W_{C}(\mathcal{F})$ and the properties of the set $\mathcal{F}$. In Sections 3 and 4 , we study conditions for $W_{C}(\mathcal{F})$ to be star-shaped or convex. In Section 5 , we consider the joint $C$-numerical range of $\left(A_{1}, \ldots, A_{k}\right) \in M_{n}^{k}$ defined by

$$
W_{C}\left(A_{1}, \ldots, A_{k}\right)=\left\{\left(\operatorname{tr}\left(C U^{*} A_{1} U\right), \ldots, \operatorname{tr}\left(C U^{*} A_{k} U\right)\right): U \text { is unitary }\right\} \subseteq \mathbb{C}^{k} .
$$

If $C, A_{1}, \ldots, A_{k}$ are Hermitian matrices, then $W_{C}\left(A_{1}, \ldots, A_{k}\right) \subseteq \mathbb{R}^{k}$. One may see [10] for the background and references on the $C$-numerical range.

If $\mu \in \mathbb{C}$ and $C=\mu I$, then

$$
W_{C}(A)=\{\mu \operatorname{tr} A\} \quad \text { and } \quad W_{C}\left(A_{1}, \ldots, A_{k}\right)=\left\{\mu\left(\operatorname{tr} A_{1}, \ldots, \operatorname{tr} A_{k}\right)\right\} .
$$

We will always assume that $C$ is not a scalar matrix to avoid trivial consideration.

For convenience of discussion, we always identify $\mathbb{C}$ with $\mathbb{R}^{2}$.

\section{Basic properties of $W_{C}(A)$ and $W_{C}(\mathcal{F})$}

We first list some basic results in the first two propositions below about the $C$-numerical range; see [4, 10, 13, 17, 18] and their references.

Proposition 2.1. Let $C, A \in M_{n}$.

(a) For any unitary $U, V \in M_{n}, W_{V^{*} C V}\left(U^{*} A U\right)=W_{C}(A)=W_{A}(C)$.

(b) For any $\alpha, \beta \in \mathbb{C}$,

$$
W_{C}(\alpha A+\beta I)=\alpha W_{C}(A)+\beta \operatorname{tr} C=\left\{\alpha \mu+\beta \operatorname{tr} C: \mu \in W_{C}(A)\right\} .
$$

(c) The set $W_{C}(A)$ is compact.

(d) The set $W_{C}(A)$ is star-shaped with $(\operatorname{tr} C)(\operatorname{tr} A) / n$ as a star center, i.e.,

$$
t \mu+(1-t)(\operatorname{tr} C)(\operatorname{tr} A) / n \in W_{C}(A) \quad \text { for any } \mu \in W_{C}(A) \text { and } t \in[0,1] \text {. }
$$

(e) The set $W_{C}(A)$ is convex if there is $\gamma \in \mathbb{C}$ such that $\tilde{C}=C-\gamma I_{n}$ satisfies any one of the following conditions.

(e.1) $\tilde{C}$ is rank one. 
(e.2) $\tilde{C}$ is a multiple of a Hermitian matrix.

(e.3) $\tilde{C}$ is unitarily similar to a block matrix of the form $\left(C_{i j}\right)_{1 \leq i, j \leq m}$ such that $C_{11}, \ldots, C_{m m}$ are square matrices possibly with different sizes, and $C_{i j}=0$ if $j \neq i+1$.

Researchers have extended the results on classical numerical range, and showed that there is interesting interplay between the geometry of $W_{C}(A)$ and the algebraic properties of $C$ and $A$. A boundary point $\nu$ of $W_{C}(A)$ is a corner point if there is $d>0$ such that $W_{C}(A) \cap\{\mu \in \mathbb{C}:|\mu-\nu| \leq d\}$ is contained in a pointed cone with $\nu$ as a vertex.

Proposition 2.2. Let $C=\left(c_{i j}\right) \in M_{n}$ be a non-scalar matrix in lower triangular form with diagonal entries $c_{1}, \ldots, c_{n}$. Suppose $A=\left(a_{i j}\right) \in M_{n}$ is also in lower triangular form with diagonal entries $a_{1}, \ldots, a_{n}$.

(a) If $\operatorname{tr}(C A)=\sum_{j=1}^{n} c_{j} a_{j}$ is a boundary point of $W_{C}(A)$, then $a_{r s}=0$ whenever $c_{r} \neq c_{s}$ and $c_{p q}=0$ whenever $a_{p} \neq a_{q}$. In particular, if $C$ and $A$ has distinct eigenvalues, then $C$ and $A$ will be in diagonal form, i.e., $C$ and $A$ are normal.

(b) If $\nu$ is a corner point of $W_{C}(A)$, then there is a unitary $V$ such that $V^{*} A V$ is lower triangular form with diagonal entries $a_{j_{1}}, \ldots, a_{j_{n}}$ such that $\left(j_{1}, \ldots, j_{n}\right)$ is a permutation of $(1, \ldots, n)$ and

$$
\nu=\operatorname{tr}\left(C V^{*} A V\right)=\sum_{\ell=1}^{n} c_{\ell} a_{j_{\ell}} .
$$

(c) The set $W_{C}(A)$ is a singleton if and only if $A$ is a scalar matrix.

(d) The set $W_{C}(A)$ is a non-degenerate line segment if and only if $C$ and $A$ are non-scalar normal matrices having collinear eigenvalues in $\mathbb{C}$.

(e) The set $W_{C}(A)$ is a convex polygon if and only if

$$
W_{C}(A)=\operatorname{conv}\left\{\left(c_{1}, \ldots, c_{n}\right) P\left(a_{1}, \ldots a_{n}\right)^{t}: P \text { is a permutation matrix }\right\} \text {. }
$$

Now, we extend the basic properties of $W_{C}(A)$ to $W_{C}(\mathcal{F})$. We always assume that $C$ is not a scalar matrix and $\mathcal{F}$ is a non-empty subset of $M_{n}$.

Theorem 2.3. Suppose $C \in M_{n}$ is non-scalar and $\mathcal{F} \subseteq M_{n}$ is non-empty.

(a) If $U \in M_{n}$ is unitary, then $W_{C}(\mathcal{F})=W_{C}\left(U^{*} \mathcal{F} U\right)$, where $U^{*} \mathcal{F} U=\left\{U^{*} A U: A \in \mathcal{F}\right\}$.

(b) For any $\alpha, \beta \in \mathbb{C}$, let $\alpha \mathcal{F}+\beta I=\{\alpha A+\beta I: A \in \mathcal{F}\}$. Then

$$
W_{C}(\alpha \mathcal{F}+\beta I)=\alpha W_{C}(\mathcal{F})+\beta \operatorname{tr} C=\left\{\alpha \mu+\beta \operatorname{tr} C: \mu \in W_{C}(\mathcal{F})\right\} .
$$


(c) If $\mathcal{F}$ is bounded, then so is $W_{C}(\mathcal{F})$.

(d) If $\mathcal{F}$ is connected, then so is $W_{C}(\mathcal{F})$.

(e) If $\mathcal{F}$ is compact, then so is $W_{C}(\mathcal{F})$.

Proof. (a) and (b) can be verified readily.

(c) If $\mathcal{F}$ is bounded so that there is $R>0$ such that for every $A \in M_{n}$ we have $\|A\|<R$, then

$$
\left|\operatorname{tr}\left(C U^{*} A U\right)\right| \leq n\|C\|\|A\|<n\|C\| R .
$$

Thus, $W_{C}(\mathcal{F})$ is bounded.

(d) Note that for any $A \in M_{n}, W_{C}(A)$ is star-shaped with $(\operatorname{tr} C)(\operatorname{tr} A) / n$ as a star center. If $\mu_{1}=\operatorname{tr}\left(C U^{*} A_{1} U\right)$ and $\mu_{2}=\operatorname{tr}\left(C V^{*} A_{2} V\right)$ with $A_{1}, A_{2} \in \mathcal{F}$ and $U, V$ unitary, then there are a line segment with end points $\mu_{1}$ and $(\operatorname{tr} C)\left(\operatorname{tr} A_{1}\right) / n$, and another line segment with end points $\mu_{2}$ and $(\operatorname{tr} C)\left(\operatorname{tr} A_{2}\right) / n$. If $\mathcal{F}$ is connected, then so are the sets $\{\operatorname{tr} A: A \in \mathcal{F}\}$ and $\{(\operatorname{tr} A)(\operatorname{tr} C) / n: A \in \mathcal{F}\}$. Hence, there is a path joining $\mu_{1}$ to $(\operatorname{tr} C)\left(\operatorname{tr} A_{1}\right) / n$, then to $(\operatorname{tr} C)\left(\operatorname{tr} A_{2}\right) / n$ and then to $\mu_{2}$.

(e) Suppose $\mathcal{F}$ is compact. Then $\mathcal{F}$ is bounded and closed. By $(\mathrm{c}), W_{C}(\mathcal{F})$ is also bounded. To show that $W_{C}(\mathcal{F})$ is closed, let $\left\{\operatorname{tr}\left(C U_{k}^{*} A_{k} U_{k}\right): k=1,2, \ldots\right\}$ be a sequence in $W_{C}(\mathcal{F})$ converging to $\mu_{0} \in \mathbb{C}$, where $A_{k} \in \mathcal{F}$ and $U_{k}$ is unitary for each $k$. Since $\mathcal{F}$ is compact, there is a subsequence $\left\{A_{j_{k}}: k=1,2, \ldots\right\}$ of $\left\{A_{k}: k=1,2, \ldots\right\}$ converging to $A_{0} \in \mathcal{F}$. We can further consider a subsequence $\left\{U_{j(\ell)}: \ell=1,2, \ldots\right\}$ of $\left\{U_{j_{k}}: k=1,2, \ldots\right\}$ converging to $U_{0}$. Thus

$$
\left\{\operatorname{tr}\left(C U_{j(\ell)}^{*} A_{j(\ell)} U_{j(\ell)}\right): k=1,2, \ldots\right\} \rightarrow \operatorname{tr}\left(C U_{0}^{*} A_{0} U_{0}\right)=\mu_{0} \in W_{C}(\mathcal{F}) .
$$

Thus, $W_{C}(\mathcal{F})$ is closed. As a result, $W_{C}(\mathcal{F})$ is compact.

The following examples show that none of the converses of the assertions in Theorem 2.3 (c) - (e) is valid, and there are no implications between the conditions that " $\mathcal{F}$ is closed" and " $W_{C}(\mathcal{F})$ " is closed.

Example 2.4. (a) Suppose $C \in M_{n}$ is non-scalar and has trace zero, and $\mathcal{F}=\{\mu I: \mu \in$ $\mathbb{C}\}$. Then $W_{C}(\mathcal{F})=\{0\}$ is bounded and compact, but $\mathcal{F}$ is not bounded.

(b) Suppose $C \in M_{n}$ is non-scalar, and $\mathcal{F}=\left\{A_{0}, A_{1}\right\}$ such that $A_{0}=0$ and $A_{1}=x y^{*}$ for a pair of orthonormal vectors $x, y$. Then $W_{C}\left(A_{0}\right)=\{0\}$ and $W_{C}(\mathcal{F})=W_{C}\left(A_{1}\right)$ is a circular disk center at the origin with radius

$$
R=\max \left\{\left|u^{*} C v\right|:\{u, v\} \text { is an orthonormal set }\right\} .
$$

Thus, $W_{C}(\mathcal{F})$ is connected, but $\mathcal{F}$ is not. 
(c) Let $\mathcal{F}=\operatorname{conv} \mathcal{G}$ with

$$
\mathcal{G}=\left\{2 E_{12}\right\} \cup\left\{\operatorname{diag}\left(e^{i r}, e^{-i r}\right): r \text { is a rational number }\right\} .
$$

Then $\mathcal{F}$ is not closed but $W(\mathcal{F})=W\left(2 E_{12}\right)$ is closed.

(d) Let $\mathcal{F}=\left\{\operatorname{diag}\left(0, x+\frac{i}{x}\right): x>0\right\} \cup\{\operatorname{diag}(0,0)\}$. Then $\mathcal{F}$ is closed, but

$$
W(\mathcal{F})=\{x+i y: x, y>0, x y \leq 1\} \cup\{0\}
$$

is not closed.

Next, we consider the connection between the geometrical properties of $W_{C}(\mathcal{F})$ and the properties of $C$ and $\mathcal{F}$. Note that for any subset $\mathcal{S}$ of $\mathbb{C}$, if $\operatorname{tr} C \neq 0$ and $\mathcal{F}=\{\mu I / \operatorname{tr} C$ : $\mu \in \mathcal{S}\}$, then we have $W_{C}(\mathcal{F})=\mathcal{S}$. Thus, the geometrical shape of $W_{C}(\mathcal{F})$ may be quite arbitrary. Also, if $C=\mu I$ is a scalar matrix, then $W_{C}(\mathcal{F})=\{\mu \operatorname{tr} A: A \in \mathcal{F}\}$. Again, $W_{C}(\mathcal{F})$ does not contain much information about the matrices in $\mathcal{F}$. Nevertheless, we have the following.

Theorem 2.5. Suppose $C \in M_{n}$ is non-scalar, and $\mathcal{F} \subseteq M_{n}$ is non-empty. The following conditions hold.

(a) The set $W_{C}(\mathcal{F})=\{\mu\}$ if and only if $\mathcal{F}=\{\nu I: \nu \operatorname{tr} C=\mu\}$.

(b) The set $W_{C}(\mathcal{F})$ is a subset of a straight line $L$ if and only if

(i) $\mathcal{F} \subseteq\{\nu I: \nu \in \mathbb{C}, \nu \operatorname{tr} C \in L\}$, or

(ii) there are complex units $\alpha, \gamma \in \mathbb{C}$ such that

$\gamma(C-(\operatorname{tr} C) I / n)$ and $\alpha(A-(\operatorname{tr} A) I / n)$ are Hermitian for all $A \in \mathcal{F}$,

and $\{(\operatorname{tr} C)(\operatorname{tr} A) / n: A \in \mathcal{F}\}$ is collinear.

(c) The set $W_{C}(\mathcal{F})$ is a convex polygon if and only if $W_{C}(\mathcal{F})=\operatorname{conv}\left\{v_{1}, \ldots, v_{m}\right\}$ where each $v_{j}$ is of the forms $\left(c_{1}, \ldots, c_{n}\right)\left(a_{1}, \ldots, a_{n}\right)^{t}$. Here $c_{1}, \ldots, c_{n}$ are eigenvalues of $C$, and $a_{1}, \ldots, a_{n}$ are eigenvalues of some $A_{j} \in \mathcal{F}$.

Proof. Condition (a) follows from the fact that $W_{C}(A)=\{\mu\}$ if and only if $A=\nu I$ with $\nu \operatorname{tr} C=\mu$.

(b) Suppose $W_{C}(\mathcal{F}) \subseteq L$. If $\mathcal{F} \subseteq\{\mu I: \mu \in \mathbb{C}\}$, then clearly $\mathcal{F} \subseteq\{\nu I: \nu \operatorname{tr} C \in L\}$. Let $\mathcal{F}$ contains a non-scalar matrix $A$. Then $W_{C}(A)$ must be a non-degenerate line segment contained in $L$. Therefore $C, A$ are normal with collinear eigenvalues in $\mathbb{C}$, see [10, (7.3)]. There exist complex units $\alpha, \gamma \in \mathbb{C}$ such that $\gamma(C-(\operatorname{tr} C) I / n)$ and $\alpha(A-(\operatorname{tr} A) I / n)$ are Hermitian. If $B \in \mathcal{F}$ is a scalar matrix, then $\alpha(B-(\operatorname{tr} B) I / n)=0$ which is Hermitian. Now assume $B \in \mathcal{F}$ is non-scalar. Let $\tilde{C}=\gamma(C-(\operatorname{tr} C) I / n)$. Then

$$
W_{\tilde{C}}(\alpha(B-(\operatorname{tr} B) I / n))=\gamma \alpha W_{C}(B)+\mu_{B} \subseteq\left\{\gamma \alpha z+\mu_{B}: z \in L\right\},
$$


for some constant $\mu_{B} \in \mathbb{C}$, and

$$
W_{\tilde{C}}(\alpha(A-(\operatorname{tr} A) I / n))=\gamma \alpha W_{C}(A)+\mu_{A} \subseteq\left\{\gamma \alpha z+\mu_{A}: z \in L\right\}
$$

for some constant $\mu_{A} \in \mathbb{C}$. Hence $W_{\tilde{C}}(\alpha(B-(\operatorname{tr} B) I / n))$ is a subset of a line segment parallel to $W_{\tilde{C}}(\alpha(A-(\operatorname{tr} A) I / n)) \subseteq \mathbb{R}$. As $0 \in W_{\tilde{C}}(\alpha(B-(\operatorname{tr} B) I / n))$, we have $W_{\tilde{C}}(\alpha(B-$ $(\operatorname{tr} B) I / n)) \subseteq \mathbb{R}$. Therefore, $\alpha(B-(\operatorname{tr} B) I / n)$ is Hermitian. The last assertion follows from $\{(\operatorname{tr} C)(\operatorname{tr} A) / n: A \in \mathcal{F}\} \subseteq W_{C}(\mathcal{F}) \subseteq L$. The sufficiency can be verified readily.

(c) Suppose $W_{C}(\mathcal{F})=\operatorname{conv}\left\{v_{1}, \ldots, v_{m}\right\}$ is a convex polygon. Then for every $v_{j}$, there is $A_{j} \in \mathcal{F}$ such that $v_{j}=\operatorname{tr}\left(C U_{j}^{*} A_{j} U_{j}\right) \in W_{C}\left(A_{j}\right)$ for some unitary $U_{j} \in M_{n}$. Since $W_{C}\left(A_{j}\right) \subseteq W_{C}(\mathcal{F})$, we see that $v_{j}$ is a vertex point of $W_{C}\left(A_{j}\right)$. It follows that $v_{j}$ has the form $\left(c_{1}, \ldots, c_{n}\right)\left(a_{1}, \ldots, a_{n}\right)^{t}$, where $c_{1}, \ldots, c_{n}$ are eigenvalues of $C$ and $a_{1}, \ldots, a_{n}$ are eigenvalues of $A_{j}$ arranged in some suitable order. The converse of the assertion is clear.

\section{Star-shapedness and Convexity}

In this section, we study the star-shapedness and convexity of $W_{C}(\mathcal{F})$. If $\mathcal{F}$ is not connected, then $W_{C}(\mathcal{F})$ may not be connected so that $W_{C}(\mathcal{F})$ is not star-shaped or convex. One might hope that if $\mathcal{F}$ is star-shaped or convex, then $W_{C}(\mathcal{F})$ will inherit the properties. However, the following examples show that $W_{C}(\mathcal{F})$ may fail to be convex (star-shaped, resp.) even if $\mathcal{F}$ is convex (star-shaped, resp.).

Example 3.1. Let $C=E_{11}, A=\operatorname{diag}(1+i, 1-i)$ and $\mathcal{F}=\operatorname{conv}\{A,-A\}$. Then

$$
W_{C}(\mathcal{F})=W(\mathcal{F})=\bigcup_{t \in[0,1]} W(t A+(1-t)(-A))=\bigcup_{s \in[-1,1]} s W(A) .
$$

As $W(A)=\operatorname{conv}\{1+i, 1-i\}$, we have $W_{C}(\mathcal{F})=\operatorname{conv}\{0,1+i, 1-i\} \cup \operatorname{conv}\{0,-1-i,-1+i\}$ which is not convex, see Figure 1 .

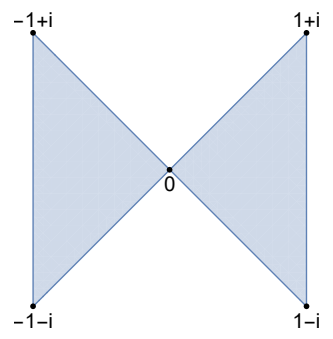

Figure 1

Example 3.2. Let $C=E_{11}, A=\operatorname{diag}(1+i, 1-i), \mathcal{F}_{1}=\operatorname{conv}\{A,-A\}$ and $\mathcal{F}_{2}=$ $\operatorname{conv}\{A,-A+4 I\}$. Then $\mathcal{F}=\mathcal{F}_{1} \cup \mathcal{F}_{2}$ is star-shaped with star-center $A$. Since $t A+$ $(1-t)(-A+4 I)=(1-2 t)(2 I-A)+2 I$, we have

$$
W\left(\mathcal{F}_{2}\right)=\bigcup_{t \in[0,1]} W((1-2 t)(-A+2 I)+2 I)=\bigcup_{s \in[-1,1]} s W(-A+2 I)+2 .
$$


Note that $W(-A+2 I)=\operatorname{conv}\{1+i, 1-i\}=W(A)$. Then $W\left(\mathcal{F}_{2}\right)=W\left(\mathcal{F}_{1}\right)+2$ and

$$
W_{C}(\mathcal{F})=W\left(\mathcal{F}_{1} \cup \mathcal{F}_{2}\right)=W\left(\mathcal{F}_{1}\right) \cup W\left(\mathcal{F}_{2}\right)
$$

equals $\operatorname{conv}\{0,-1+i,-1-i\} \cup \operatorname{conv}\{0,2,1-i, 1+i\} \cup \operatorname{conv}\{2,3+i, 3-i\}$ which is not star-shaped, see Figure 2.

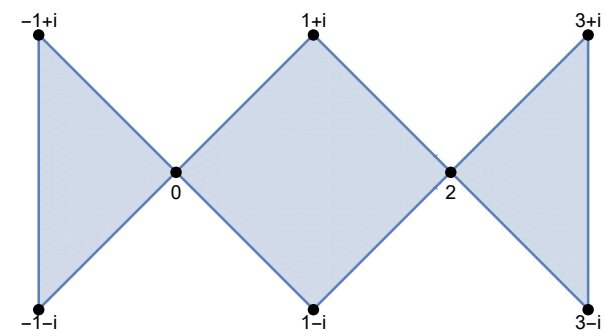

Figure 2

Notice that in Example $3.1, \mathcal{F}$ is convex and $W_{C}(\mathcal{F})$ is star-shaped with star-center at the origin. One may ask if $W_{C}(\mathcal{F})$ is always star-shaped for a convex set $\mathcal{F}$. We will study this question in the following, and pay special attention on the case where $\mathcal{F}=\operatorname{conv}\left\{A_{1}, \ldots, A_{m}\right\}$ for some $A_{1}, \ldots, A_{m} \in M_{n}$.

Denote by $S_{C}(A)$ the set of all star-centers of $W_{C}(A)$ and $\mathcal{U}_{n}$ the group of all $n \times n$ unitary matrices. We begin with the following result showing that $W_{C}(\mathcal{F})$ is star-shaped if $C$ or $\mathcal{F}$ satisfies some special properties.

Proposition 3.3. Suppose $C \in M_{n}$ and $\mathcal{F}$ is a convex matrix set.

(a) If $\mathcal{F}$ contains a scalar matrix $\mu I$, then $W_{C}(\mathcal{F})$ is star-shaped with $\mu \operatorname{tr} C$ as a star center.

(b) Suppose the intersection of all (or any three of) $S_{C}(A)$ with $A \in \mathcal{F}$ is nonempty. Then $W_{C}(\mathcal{F})$ is star-shaped with $\mu$ as a star center for any $\mu \in \cap\left\{S_{C}(A): A \in \mathcal{F}\right\}$.

(c) If $\operatorname{tr} C=0$, then $W_{C}(\mathcal{F})$ is star-shaped with 0 as a star-center.

(d) If all matrices in $\mathcal{F}$ have the same trace $\nu$, then $W_{C}(\mathcal{F})$ is star-shaped with $\nu \operatorname{tr} C$ as a star-center.

Proof. (a) Suppose $\mathcal{F}$ contains a scalar matrix $\mu I$ and $B \in \mathcal{F}$. Then

$$
\operatorname{conv}\left\{\mu \operatorname{tr} C, W_{C}(B)\right\} \subseteq W_{C}(\operatorname{conv}\{\mu I, B\}) \subseteq W_{C}(\mathcal{F})
$$

The result follows.

(b) Suppose $\mu \in \cap\left\{S_{C}(A): A \in \mathcal{F}\right\}$. Then for any $\nu \in W_{C}(\mathcal{F})$, there is $B \in \mathcal{F}$ such that $\nu \in W_{C}(B)$. As $\mu \in S_{C}(B)$, the line segment joining $\mu$ and $\nu$ will lie in $W_{C}(B) \subseteq W_{C}(\mathcal{F})$. Thus, $W_{C}(\mathcal{F})$ is star-shaped with $\mu$ as a star center. 
If $S_{C}\left(A_{0}\right) \cap S_{C}\left(A_{1}\right) \cap S_{C}\left(A_{2}\right) \neq \emptyset$ for any $A_{0}, A_{1}, A_{2} \in \mathcal{F}$, then $\cap\left\{S_{C}(A): A \in \mathcal{F}\right\} \neq \emptyset$ by Helly's Theorem. So, the result follows from the preceding paragraph.

(c) Note that $\frac{1}{n}(\operatorname{tr} C)(\operatorname{tr} A) \in S_{C}(A)$ for any $C, A \in M_{n}$, see [4] or Proposition 2.1 (d). If $\operatorname{tr} C=0$, then $0 \in \bigcap\left\{S_{C}(A): A \in \mathcal{F}\right\}$ is a star-center of $W_{C}(\mathcal{F})$ by (b).

(d) The assumption implies that $\nu \operatorname{tr} C$ is the common star-center of $W_{C}(A)$ for all $A \in \mathcal{F}$. Thus, the result follows from (b).

Lemma 3.4. Let $C, A, B \in M_{n}$ and $\mathcal{F}=\operatorname{conv}\{A, B\}$. If $\mu \in S_{C}(A) \cap S_{C}(B)$, then $W_{C}(\mathcal{F})$ is star-shaped with star-center $\mu$.

Proof. Let $\zeta \in W_{C}(\mathcal{F})$. There are $V \in \mathcal{U}_{n}$ and $0 \leq t \leq 1$ such that $\zeta=\operatorname{tr}\left(C V^{*}(t A+(1-\right.$ t) $B) V)$. It suffices to show $\operatorname{conv}\left\{\mu, \operatorname{tr}\left(C V^{*} A V\right), \operatorname{tr}\left(C V^{*} B V\right)\right\} \subseteq W_{C}(\mathcal{F})$. For any $a, b, c \in \mathbb{C}$, we let $\triangle(a, b, c)=\operatorname{conv}\{a, b\} \cup \operatorname{conv}\{b, c\} \cup \operatorname{conv}\{a, c\}$, i.e., the triangle (without the interior) with the vertices $a, b, c$. Let $U_{A} \in \mathcal{U}_{n}$ such that $\operatorname{tr}\left(C U_{A}^{*} A U_{A}\right)=\mu$. As $\mu \in S_{C}(A) \cap S_{C}(B)$ we have

$$
\operatorname{conv}\left\{\operatorname{tr}\left(C V^{*} A V\right), \mu\right\} \cup \operatorname{conv}\left\{\operatorname{tr}\left(C V^{*} B V\right), \mu\right\} \subseteq W_{C}(\mathcal{F}) .
$$

Moreover we have

$$
\operatorname{conv}\left\{\operatorname{tr}\left(C V^{*} A V\right), \operatorname{tr}\left(C V^{*} B V\right)\right\}=\left\{\operatorname{tr}\left(C V^{*}(t A+(1-t) B) V\right): 0 \leq t \leq 1\right\} \subseteq W_{C}(\mathcal{F}) .
$$

Hence $\triangle\left(\operatorname{tr}\left(C V^{*} A V\right), \operatorname{tr}\left(C V^{*} B V\right), \mu\right) \subseteq W_{C}(\mathcal{F})$. We shall show that

$$
\operatorname{conv}\left\{\operatorname{tr}\left(C V^{*} A V\right), \operatorname{tr}\left(C V^{*} B V\right), \mu\right\} \subseteq W_{C}(\mathcal{F}) .
$$

If $\triangle\left(\operatorname{tr}\left(C V^{*} A V\right), \operatorname{tr}\left(C V^{*} B V\right), \mu\right)$ is a line segment or a point, then eq. (3.1) holds clearly. Now assume that $\triangle\left(\operatorname{tr}\left(C V^{*} A V\right), \operatorname{tr}\left(C V^{*} B V\right), \mu\right)$ is non-degenerate. As $\mathcal{U}_{n}$ is path-connected, we define a continuous function $f:[0,1] \rightarrow \mathcal{U}_{n}$ with $f(0)=V$ and $f(1)=U_{A}$. For $0 \leq t \leq 1$, let $V_{A}(t)=\operatorname{tr}\left(C f(t)^{*} A f(t)\right)$ and $V_{B}(t)=\operatorname{tr}\left(C f(t)^{*} B f(t)\right)$. Note that for any $t \in[0,1]$, we have

$$
\triangle(t)=\triangle\left(V_{A}(t), V_{B}(t), \mu\right) \subseteq W_{C}(\mathcal{F}) .
$$

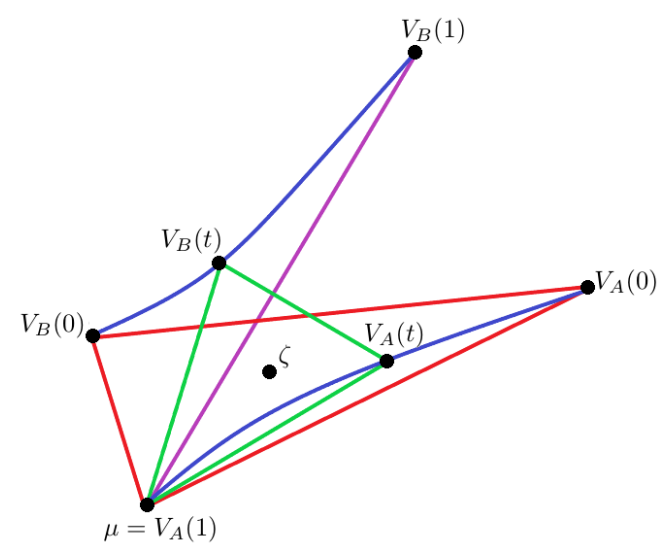

Figure 3: $\triangle(0)$ and $\triangle(t)$ are triangles in the red and green, respectively. In particular, $\triangle(1)$ dengenerates to a line segment in purple. 
For any $\zeta \in \operatorname{conv} \triangle(0)$, see Figure 3 , let

$$
t_{0}=\max \{t: \zeta \in \operatorname{conv} \triangle(s) \text { for all } 0 \leq s \leq t\}
$$

Since $\triangle(1)$ degenerates, by continuity of $f$, we have $\zeta \in \triangle\left(t_{0}\right) \subseteq W_{C}(\mathcal{F})$. Hence the result follows.

One can extend Lemma 3.4 to a more general situation.

Theorem 3.5. Let $C \in M_{n}$ and $\mathcal{G}$ be a (finite or infinite) family of matrices in $M_{n}$. If $\mu \in \bigcap_{A \in \mathcal{G}} S_{C}(A)$, then $W_{C}(\mathcal{F})$ is star-shaped with star-center $\mu$ for $\mathcal{F}=$ conv $\mathcal{G}$.

Proof. By Lemma 3.4, the result holds if $\mathcal{G}$ has 2 elements. Suppose $|\mathcal{G}| \geq 3$ and $\mu \in$ $\bigcap_{A \in \mathcal{G}} S_{c}(A)$. Let $\zeta \in W_{C}(\mathcal{F})$. Then there exist $A_{1}, \ldots, A_{m} \in \mathcal{G}, t_{1}, \ldots, t_{m}>0$ with $t_{1}+\cdots+$ $t_{m}=1$ and $U \in \mathcal{U}_{n}$ such that $\zeta=\operatorname{tr}\left(C U^{*}\left(t_{1} A_{1}+\cdots+t_{m} A_{m}\right) U\right)$. Let $\zeta_{i}=\operatorname{tr}\left(C U^{*} A_{i} U\right)$, $i=1, \ldots, m$. Then $\zeta \in \operatorname{conv}\left\{\zeta_{1}, \ldots, \zeta_{m}\right\}$. The half line from $\mu$ through $\zeta$ intersects a line segment joining some $\zeta_{i}, \zeta_{j}$ with $1 \leq i \leq j \leq m$ such that $\zeta \in \operatorname{conv}\left\{\mu, \zeta_{i}, \zeta_{j}\right\}$. By Lemma 3.4. we have $\operatorname{conv}\left\{\mu, \zeta_{i}, \zeta_{j}\right\} \subseteq W_{C}\left(\operatorname{conv}\left\{A_{i}, A_{j}\right\}\right) \subseteq W_{C}(\mathcal{F})$.

Note that if $W_{C}(A)$ is convex, then $W_{C}(A)=S_{C}(A)$. So, if $W_{C}(A)$ is convex for every $A \in \mathcal{G}$, and if $\mu \in \cap_{A \in \mathcal{G}} W_{C}(A)$, then $\mu$ is a star-center of $W_{C}(\operatorname{conv}(\mathcal{G}))$. Checking the condition that $W_{C}(A)$ is convex for every $A \in \mathcal{G}$ may not be easy. On the other hand, if $C \in M_{n}$ is such that $W_{C}(A)$ is always convex, then one can skip the checking process, and we have the following.

Corollary 3.6. Suppose $C \in M_{n}$ satisfies any one of the conditions (e.1)-(e.3) in Proposition 2.1 (e). If $\mathcal{G} \subseteq M_{n}$ and $\mu \in \cap_{A \in \mathcal{G}} W_{C}(A)$, then $\mu$ is a star-center of $W_{C}($ convG).

Next, we show that if $W_{C}(A)$ and $W_{C}(B)$ are convex, then $W_{C}(\mathcal{F})$ is star-shaped for $\mathcal{F}=\operatorname{conv}\{A, B\}$ even when $W_{C}(A) \cap W_{C}(B)=\emptyset$.

Theorem 3.7. Let $C \in M_{n}$. Suppose $A, B \in M_{n}$ such that $W_{C}(A)$ and $W_{C}(B)$ are convex sets with empty intersection. Let $\mathcal{F}=\operatorname{conv}\{A, B\}$.

If $W_{C}(A) \cup W_{C}(B)$ lies on a line, then $W_{C}(\mathcal{F})=\operatorname{conv}\left\{W_{C}(A), W_{C}(B)\right\}$ is convex.

Otherwise, there are two non-parallel lines $L_{1}$ and $L_{2}$ intersecting at $\mu$ such that for each $j=1,2, L_{j}$ is a common supporting line of $W_{C}(A)$ and $W_{C}(B)$ separating the two convex sets (i.e., $W_{C}(A)$ and $W_{C}(B)$ lying on opposite closed half spaces determined by $L_{j}$ ); the set $W_{C}(\mathcal{F})$ is star-shaped with star-center $\mu$, see Figure 4 . 


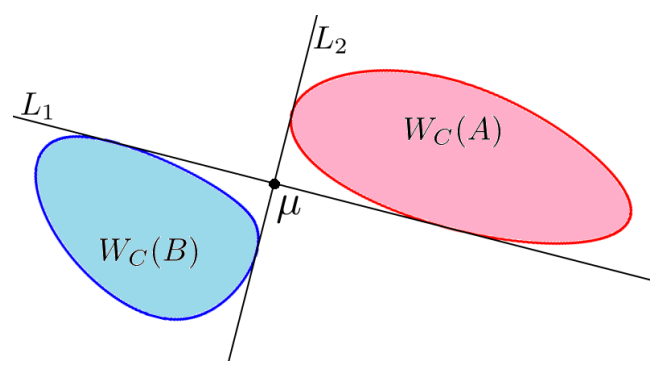

Figure 4

Proof. Assume $W_{C}(A) \cup W_{C}(B)$ lies on a line. As $\mathcal{F}$ is connected, by Theorem 2.3, $W_{C}(\mathcal{F})$ is connected. We have

$$
W_{C}(A) \cup W_{C}(B) \subseteq W_{C}(\mathcal{F}) \subseteq \operatorname{conv}\left\{W_{C}(A), W_{C}(B)\right\}
$$

Therefore, $W_{C}(\mathcal{F})=\operatorname{conv}\left\{W_{C}(A), W_{C}(B)\right\}$ which is convex.

Otherwise, we may assume that $W_{C}(A)$ lies on the left open half plane and $W_{C}(B)$ lies on the right open half plane. For $t \in[-\pi, \pi]$, let $L(t)$ be the support line of $W_{C}(A)$ with (outward pointing) normal $n(t)=(\cos t, \sin t)$. Let $P(t)=L(t) \cap W_{C}(A)$. Therefore, for each $t \in[-\pi, \pi]$ and $p \in P(t)$, we have $n(t) \cdot(w-p) \leq 0$ for all $w \in W_{C}(A)$. Let $q(t)=$ $\min \left\{n(t) \cdot(w-p): w \in W_{C}(B), p \in P(t)\right\}$. Then we have $q(0)>0$ and $q(-\pi)=q(\pi)<0$. Hence, there exist $-\pi<t_{1}<0<t_{2}<\pi$ such that $q\left(t_{1}\right)=q\left(t_{2}\right)=0$. Then $L_{j}=L\left(t_{j}\right)$ for $j=1,2$ will satisfy the requirement.

We claim that $L\left(t_{1}\right)$ and $L\left(t_{2}\right)$ in (a) cannot be parallel. Otherwise, we must have $t_{1}=t_{2}+\pi$. Therefore, $n\left(t_{1}\right)=-n\left(t_{2}\right)$ and $W_{C}(A), W_{C}(B) \subset L_{1}=L_{2}$, is a contradiction. Since $L_{1}$ and $L_{2}$ are not parallel, they intersect at a point $\mu$.

Now, we show that $\mu$ is a star-center of $W_{C}(\mathcal{F})$. We may apply a suitable affine transform to $A$ and $B$, and assume that $\mu=0, L_{1}$ and $L_{2}$ are the $x$-axis and $y$-axis respectively. We may further assume that $W_{C}(A)$ lies in the first quadrant and $W_{C}(B)$ lies in the third quadrant. For any $\zeta \in W_{C}(\mathcal{F})$, there are $V \in \mathcal{U}_{n}$ and $0 \leq t \leq 1$ such that $\zeta=\operatorname{tr}\left(C V^{*}(t A+(1-t) B) V\right)$. Denote $\zeta_{A}=\operatorname{tr}\left(C V^{*} A V\right)$ and $\zeta_{B}=\operatorname{tr}\left(C V^{*} B V\right)$. We claim that $\operatorname{conv}\left\{\zeta_{A}, \zeta_{B}, 0\right\} \subseteq W_{C}(\mathcal{F})$. Once the claim holds, we have $\{s \zeta+(1-s) \cdot 0: 0 \leq s \leq 1\} \subseteq \operatorname{conv}\left\{\zeta_{A}, \zeta_{B}, 0\right\} \subseteq W_{C}(\mathcal{F})$. Then the star-shapedness of $W_{C}(\mathcal{F})$ follows.

We now show the claim. We denote by $\overline{\zeta_{1} \zeta_{2}}$ the line segment with end points $\zeta_{1}, \zeta_{2} \in \mathbb{C}$. By symmetry, we may assume that the line segment $\overline{\zeta_{A} \zeta_{B}}$ intersects the $y$-axis at $(0, b)$ with $b \geq 0$. The situation is depicted in Figure $5 \mathrm{a}$ and Figure $5 \mathrm{~b}$.

We shall first show that $\operatorname{conv}\left\{\zeta_{B}, b i, 0\right\} \subseteq W_{C}(\mathcal{F})$. Let $y_{B}$ be a point in the intersection of $W_{C}(B)$ and the $y$-axis, $U_{B} \in \mathcal{U}_{n}$ be such that $y_{B}=\operatorname{tr}\left(C U_{B}^{*} B U_{B}\right)$. Let $y_{A}=\operatorname{tr}\left(C U_{B}^{*} A U_{B}\right)$. Then by the convexity of $W_{C}(A), W_{C}(B)$ and $\mathcal{F}$, we have

$$
Q\left(\left(\zeta_{A}, y_{A}\right) ;\left(\zeta_{B}, y_{B}\right)\right):=\overline{\zeta_{A} y_{A}} \cup \overline{\zeta_{B} y_{B}} \cup \overline{\zeta_{A} \zeta_{B}} \cup \overline{y_{A} y_{B}} \subseteq W_{C}(\mathcal{F})
$$




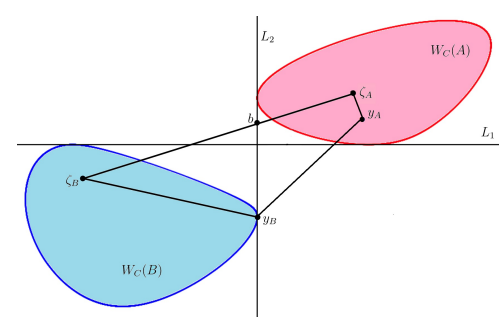

(a) $Q\left(\left(\zeta_{A}, y_{A}\right) ;\left(\zeta_{B}, y_{B}\right)\right)$ is a quadrilateral.

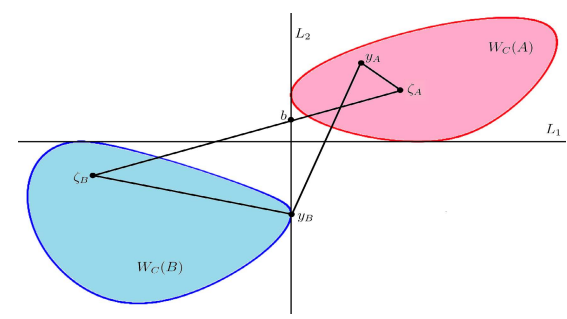

(b) $Q\left(\left(\zeta_{A}, y_{A}\right) ;\left(\zeta_{B}, y_{B}\right)\right)$ is a union of two triangle.

Figure 5

Note that $Q\left(\left(\zeta_{A}, y_{A}\right) ;\left(\zeta_{B}, y_{B}\right)\right)$ is either a quadrilateral or a union of two triangle, see Figure $5 \mathrm{a}$ and Figure $5 \mathrm{~b}$. In both case, $\triangle\left(\zeta_{B}, b i, 0\right)$ lies inside the region determined by the closed curve $Q\left(\left(\zeta_{A}, y_{A}\right) ;\left(\zeta_{B}, y_{B}\right)\right)$. Now consider a continuous function $f:[0,1] \rightarrow \mathcal{U}_{n}$ with $f(0)=V$ and $f(1)=U_{B}$. Then for $t \in[0,1]$, let $\zeta_{A}^{\prime}(t)=\operatorname{tr}\left(C f(t)^{*} A f(t)\right)$ and $\zeta_{B}^{\prime}(t)=\operatorname{tr}\left(C f(t)^{*} B f(t)\right)$, we have

$$
Q\left(\left(\zeta_{A}^{\prime}(t), y_{A}\right) ;\left(\zeta_{B}^{\prime}(t), y_{B}\right)\right):=\overline{\zeta_{A}^{\prime}(t) y_{A}} \cup \overline{\zeta_{B}^{\prime}(t) y_{B}} \cup \overline{\zeta_{A}^{\prime}(t) \zeta_{B}^{\prime}(t)} \cup \overline{y_{A} y_{B}} \subseteq W_{C}(\mathcal{F}) .
$$

Note that $Q\left(\left(\zeta_{A}^{\prime}(1), y_{A}\right) ;\left(\zeta_{B}^{\prime}(1), y_{B}\right)\right)$ degenerates, by continuity of $f$, for any point $\zeta$ enclosed by the closed curve $Q\left(\left(\zeta_{A}, y_{A}\right) ;\left(\zeta_{B}, y_{B}\right)\right)$, there exists $0 \leq t_{0} \leq 1$ such that $\zeta \in$ $Q\left(\left(\zeta_{A}^{\prime}\left(t_{0}\right), y_{A}\right) ;\left(\zeta_{B}^{\prime}\left(t_{0}\right), y_{B}\right)\right) \subseteq W_{C}(\mathcal{F})$. Hence, $\operatorname{conv}\left\{\zeta_{B}, b i, 0\right\} \subseteq W_{C}(\mathcal{F})$. By symmetry, one can show that $\operatorname{conv}\left\{\zeta_{A}, a, 0\right\} \subseteq W_{C}(\mathcal{F})$ where $a$ is the intersection point of $\overline{\zeta_{A} \zeta_{B}}$ and $x$-axis. Hence, $\operatorname{conv}\left\{\zeta_{A}, \zeta_{B}, 0\right\}=\operatorname{conv}\left\{\zeta_{A}, a, 0\right\} \cup \operatorname{conv}\left\{\zeta_{B}, b i, 0\right\} \subseteq W_{C}(\mathcal{F})$. The claim follows.

\section{Additional results on star-shapedness and convexity}

It is not easy to extend Theorem 3.7. The following example shows that if $W_{C}(A)$ and $W_{C}(B)$ are not convex, then $W_{C}(\mathcal{F})$ may not be star-shaped for $\mathcal{F}=\operatorname{conv}\{A, B\}$ even when $W_{C}(A) \cap W_{C}(B) \neq \emptyset$.

Example 4.1. Let $w=e^{2 \pi i / 3}$ and $C=\operatorname{diag}\left(1, w, w^{2}\right)$. Suppose $A=C-\frac{1}{6} I, B=e^{\pi i / 3} C+\frac{1}{6} I$ and $\mathcal{F}=\operatorname{conv}\{A, B\}$. Then $W_{C+I}(\mathcal{F})$ is not star-shaped.

To prove our claim, for $0 \leq t \leq 1$, let

$$
\begin{aligned}
& A(t)=t A+(1-t) B=\left(t+(1-t) e^{\pi i / 3}\right) C+\frac{1-2 t}{6} I . \\
W_{C+I}(A(t))= & W_{C+I}\left(\left(t+(1-t) e^{\pi i / 3}\right) C+\frac{1-2 t}{6} I\right) \\
= & \left(t+(1-t) e^{\pi i / 3}\right) W_{C}(C)+\left(t+(1-t) e^{\pi i / 3}+\frac{1-2 t}{6}\right) \operatorname{tr} C+\frac{1-2 t}{2} \\
= & \left(t+(1-t) e^{\pi i / 3}\right) W_{C}(C)+\frac{1-2 t}{2} .
\end{aligned}
$$


Therefore,

$$
W_{C+I}(\mathcal{F})=\bigcup\left\{\left(t+(1-t) e^{\pi i / 3}\right) W_{C}(C)+\frac{1-2 t}{2}: 0 \leq t \leq 1\right\} .
$$

By [14], $W_{C}(C)$ is star-shaped with the origin as the unique star-center. Moreover $W_{C}(C)=e^{2 \pi i / 3} W_{C}(C)$ and its boundary is given by

$$
\Gamma=\left\{2 e^{i \theta}+e^{-2 i \theta}:-\pi \leq \theta \leq \pi\right\}
$$

Hence, $W_{C+I}(A(t))$ is star-shaped with $\frac{1-2 t}{2}$ as the unique star-center and its boundary is given by

$$
\Gamma(t)=\left\{\left(t+(1-t) e^{\pi i / 3}\right)\left(2 e^{i \theta}+e^{-2 i \theta}\right):-\pi \leq \theta \leq \pi\right\} .
$$

For $0 \leq t \leq 1$ and $-\pi \leq \theta \leq \pi$, define

$$
\begin{aligned}
f(\theta, t)= & \left(t+(1-t) e^{\pi i / 3}\right)\left(2 e^{i \theta}+e^{-2 i \theta}\right)+\frac{1-2 t}{2} \\
= & \frac{1+t}{2}(2 \cos \theta+\cos 2 \theta)-\frac{1-t}{2} \sqrt{3}(2 \sin \theta-\sin 2 \theta)+\frac{1-2 t}{2} \\
& +i\left(\frac{1-t}{2} \sqrt{3}(2 \cos \theta+\cos 2 \theta)+\frac{1+t}{2}(2 \sin \theta-\sin 2 \theta)\right) .
\end{aligned}
$$

Using this description, we can plot $W_{C+I}(\mathcal{F})$ as follows.

First consider the plot of $\Gamma(0)$ and $\Gamma(1)$, which are the boundaries of $W_{C+I}(B)$ and $W_{C+I}(A)$ respectively, see Figure 6 .

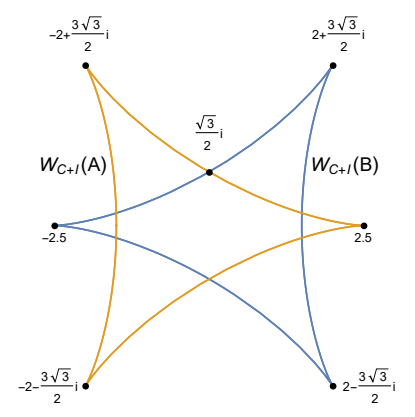

Figure 6: $W_{C+I}(A) \cup W_{C+I}(B)$

The "vertices" of $\Gamma(0)$ and $\Gamma(1)$ are given by

$$
\begin{array}{llll}
f\left(-\frac{2 \pi}{3}, 0\right)=2-\frac{3 \sqrt{3}}{2} i & f(0,0)=2+\frac{3 \sqrt{3}}{2} i & f\left(\frac{2 \pi}{3}, 0\right)=-\frac{5}{2} \\
f\left(-\frac{2 \pi}{3}, 1\right)=-2-\frac{3 \sqrt{3}}{2} i & f(0,1)=\frac{5}{2} & f\left(\frac{2 \pi}{3}, 1\right)= & -2+\frac{3 \sqrt{3}}{2} i .
\end{array}
$$

As $t$ increases from 0 to $1, \Gamma(t)$ changes from $W_{C+I}(B)$ to $W_{C+I}(A)$, For $\theta=-\frac{2 \pi}{3}, 0, \frac{2 \pi}{3}$, the vertex $f(\theta, t)$ moves along the line segment from $f(\theta, 0)$ to $f(\theta, 1)$, see Figure 7 . 


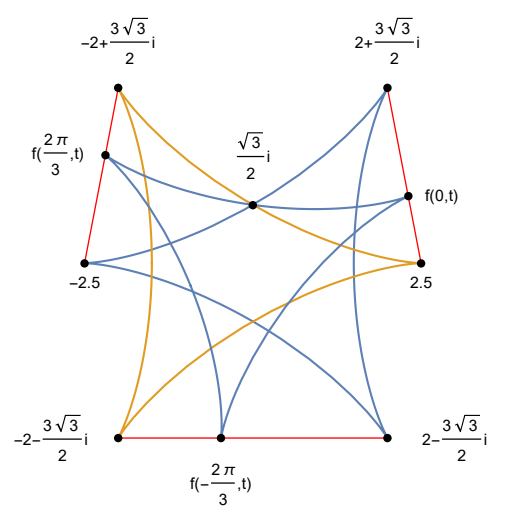

Figure 7: $W_{C+I}(\mathcal{F})$

Therefore, we see that $W_{C+I}(\mathcal{F})$ is the union of $W_{C+I}(A), W_{C+I}(B)$ and three triangles, $\triangle\left(0.5,2.5,2+\frac{3 \sqrt{3}}{2} i\right), \triangle\left(-0.5,-2.5,-2+\frac{3 \sqrt{3}}{2} i\right), \triangle\left(\frac{\sqrt{3}}{2} i, 2-\frac{3 \sqrt{3}}{2} i,-2-\frac{3 \sqrt{3}}{2} i\right)$, see Figure 8 .

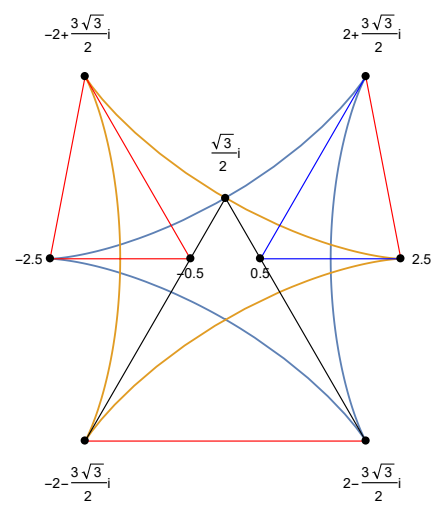

Figure 8: $W_{C+I}(\mathcal{F})$

Suppose $W_{C+I}(\mathcal{F})$ is star-shaped. By symmetry, $W_{C+I}(\mathcal{F})$ has a star-center $c$ on the imaginary axis. The line segment joining $c$ and $f(0,0)$ must lie below the tangent line to $\Gamma(0)$ at $f(0,0)=2+\frac{3 \sqrt{3}}{2} i$. By direct calculation, this tangent line is the line through 0.5 and $f(0,0)$. Similarly, The line segment joining $c$ and $f(1,0)$ must lie above the tangent line to $\Gamma(1)$ at $f(1,0)=2.5$. By direct calculation, this tangent line is the line through 0.5 and $f(1,0)$. Since these two tangent lines intersect at 0.5 , no $c$ on the imaginary axis will satisfy the above conditions.

In the following, we present an example of $C, A_{1}, A_{2}, A_{3} \in M_{n}$ such that $W_{C}\left(A_{1}\right), W_{C}\left(A_{2}\right)$, $W_{C}\left(A_{3}\right)$ are convex and $W_{C}\left(A_{1}\right) \cap W_{C}\left(A_{2}\right) \cap W_{C}\left(A_{3}\right)=\emptyset$, but $W_{C}(\mathcal{F})$ is not star-shaped for $\mathcal{F}=\operatorname{conv}\left\{A_{1}, A_{2}, A_{3}\right\}$. In particular, we choose $C=E_{11}$ so that $W_{C}(\mathcal{F})=W(\mathcal{F})$.

To describe our example, we first show that the set $W(\mathcal{F})$ is connected to the concept of product numerical range arising in the study of quantum information theory, [15]. 
Let $A \in M_{m} \otimes M_{n}=M_{m}\left(M_{n}\right)$, the product numerical range of $A$ is

$$
W^{\otimes}(A)=\left\{(u \otimes v)^{*} A(u \otimes v): u \in \mathbb{C}^{m}, v \in \mathbb{C}^{n}\right\}
$$

We note that $W^{\otimes}(A)$ depends on order of the factors $M_{m}$ and $M_{n}$ in the representation of $M_{m n}=M_{m} \otimes M_{n}$.

Theorem 4.2. Let $A_{1}, \ldots, A_{m} \in M_{n}$ and $\mathcal{F}=\operatorname{conv}\left(\left\{A_{1}, \ldots, A_{m}\right\}\right)$. Then

$$
W(\mathcal{F})=W^{\otimes}\left(\oplus_{i=1}^{m} A_{i}\right) .
$$

Proof. Let $\mu \in W(\mathcal{F})$ Then there exist $t_{1}, \ldots, t_{m} \geq 0$ with $\sum_{i=1}^{m} t_{i}=1$ and $v \in \mathbb{C}^{n}$ such that $\mu=v^{*}\left(\sum_{i=1}^{m} t_{i} A_{i}\right) v$. Let $u=\left(\sqrt{t_{1}}, \ldots, \sqrt{t_{m}}\right)^{t}$. Then $\mu=(u \otimes v)^{*}\left(\oplus_{i=1}^{m} A_{i}\right)(u \otimes v) \in$ $W^{\otimes}\left(\oplus_{i=1}^{m} A_{i}\right)$.

Conversely, suppose $\mu \in W^{\otimes}\left(\oplus_{i=1}^{m} A_{i}\right)$. Then there exist unit vectors $u \in \mathbb{C}^{m}, v \in \mathbb{C}^{n}$ such that $\mu=(u \otimes v)^{*}\left(\oplus_{i=1}^{m} A_{i}\right)(u \otimes v)$. Let $u=\left(u_{1}, \ldots, u_{m}\right)^{t}$. Set $t_{i}=\left|u_{i}\right|^{2}$ for $i=1, \ldots, m$. Then $\mu=v^{*}\left(\sum_{i=1}^{m} t_{i} A_{i}\right) v \in W(\mathcal{F})$.

Now, we describe the follow example showing that $W(\mathcal{F})$ is not necessarily star-shaped for $\mathcal{F}=\operatorname{conv}\left\{A_{1}, A_{2}, A_{3}\right\}$ if $W\left(A_{1}\right) \cap W\left(A_{2}\right) \cap W\left(A_{3}\right)=\emptyset$.

Example 4.3. Suppose $A=\operatorname{diag}\left(e^{i \frac{\pi}{3}}, e^{-i \frac{\pi}{3}}, 0.95 e^{i \frac{\pi}{4}}\right)$. Let $A_{1}=e^{i \frac{\pi}{3}} A, A_{2}=e^{-i \frac{\pi}{3}} A$ and $A_{3}=0.95 e^{i \frac{\pi}{4}} A$. Then by Theorem 4.2 , $W\left(\operatorname{conv}\left\{A_{1}, A_{2}, A_{3}\right\}\right)$ is equal to

$$
W^{\otimes}\left(\oplus_{i=1}^{m} A_{i}\right)=W^{\otimes}(A \otimes A)=W(A) \cdot W(A)=\left\{\mu_{1} \mu_{2}: \mu_{1}, \mu_{2} \in W(A)\right\} .
$$

By the result in [11, Example 3.1], $W(A) \cdot W(A)$ is not star-shaped.

We note that in this example, $W\left(A_{i}\right) \cap W\left(A_{j}\right) \neq \emptyset$ for all $i, j$, but $W\left(A_{1}\right) \cap W\left(A_{2}\right) \cap$ $W\left(A_{3}\right)=\emptyset$. Therefore, in some sense, the condition in Theorem 3.5 is optimal.

While Theorem 3.5 and Theorem 3.7 provide some sufficient conditions for the starshapedness of $W_{C}(\mathcal{F})$, it is a challenging problem to determine whether $W_{C}(\mathcal{F})$ is star-shaped or not for a given $C \in M_{n}$ and $\mathcal{F} \subseteq M_{n}$.

Proposition 4.4. Suppose $\mathcal{F}=\operatorname{conv}\left\{A_{1}, \ldots, A_{m}\right\} \subseteq M_{n}$. For each unit vector $x \in \mathbb{C}^{n}$, let $\mathcal{A}_{x}=\operatorname{diag}\left(x^{*} A_{1} x, \ldots, x^{*} A_{m} x\right)$, and let $\hat{\mathcal{F}}=\left\{\mathcal{A}_{x}: x \in \mathbb{C}^{n}, x^{*} x=1\right\}$. Then

$$
W(\mathcal{F})=W(\hat{\mathcal{F}})=W(\operatorname{conv} \hat{\mathcal{F}}) .
$$

Proof. Let $\mu \in W(\mathcal{F})$. Then there exist $t_{1}, \ldots, t_{m} \geq 0, t_{1}+\cdots+t_{m}=1$ and a unit vector $x \in \mathbb{C}^{n}$ such that $\mu=x^{*}\left(t_{1} A_{1}+\cdots+t_{m} A_{m}\right) x=t_{1} x^{*} A_{1} x+\cdots+t_{m} x^{*} A_{m} x \in W(\hat{\mathcal{F}})$. Therefore, $W(\mathcal{F}) \subseteq W(\hat{\mathcal{F}})$. 
Clearly, $W(\hat{\mathcal{F}}) \subseteq W(\operatorname{conv} \hat{\mathcal{F}})$. Finally, we show that $W(\operatorname{conv} \hat{\mathcal{F}}) \subseteq W(\mathcal{F})$. To see this, let $\mu \in W(\operatorname{conv} \hat{\mathcal{F}})$, i.e.,

$$
\mu=y^{*}\left(\sum_{j=1}^{r} t_{j} D_{j}\right) y
$$

for a unit vector $y=\left(y_{1}, \ldots, y_{m}\right)^{t}$, some $D_{j}=\operatorname{diag}\left(x_{j}^{*} A_{1} x_{j}, \ldots, x_{j}^{*} A_{m} x_{j}\right) \in \hat{\mathcal{F}}$ with $j=$ $1, \ldots, r$ and $t_{1}, \ldots, t_{r}>0, t_{1}+\cdots+t_{r}=1$. Thus,

$$
\begin{aligned}
\mu & =\sum_{\ell=1}^{m}\left|y_{\ell}\right|^{2} \sum_{j=1}^{r} t_{j} x_{j}^{*} A_{\ell} x_{j}=\sum_{j=1}^{r} t_{j}\left(x_{j}^{*}\left(\sum_{\ell=1}^{m}\left|y_{\ell}\right|^{2} A_{\ell}\right) x_{j}\right) \\
& \in \operatorname{conv}\left(W\left(A_{0}\right)\right)=W\left(A_{0}\right) \subseteq W(\mathcal{F})
\end{aligned}
$$

where $A_{0}=\sum_{\ell=1}^{m}\left|y_{\ell}\right|^{2} A_{\ell} \in \mathcal{F}$.

Example 4.5. Suppose that $A_{1}=\operatorname{diag}\left(1, e^{i \frac{\pi}{3}}\right), A_{2}=\operatorname{diag}\left(e^{i \frac{2 \pi}{3}}, e^{i \pi}\right), A_{2}=\operatorname{diag}\left(e^{i \frac{4 \pi}{3}}, e^{i \frac{5 \pi}{3}}\right)$ and $\mathcal{F}=\operatorname{conv}\left\{A_{1}, A_{2}, A_{3}\right\}$. Then $W\left(A_{1}\right) \cap W\left(A_{2}\right) \cap W\left(A_{3}\right)=\emptyset$. So the condition in Theorem 3.5 is not satisfied. However, by direct computation, we have

$$
\hat{\mathcal{F}}=\left\{\operatorname{diag}\left(t+(1-t) e^{i \frac{\pi}{3}}, e^{i \frac{2 \pi}{3}}\left(t+(1-t) e^{i \frac{\pi}{3}}\right), e^{i \frac{4 \pi}{3}}\left(t+(1-t) e^{i \frac{\pi}{3}}\right)\right): 0 \leq t \leq 1\right\}
$$

where $\hat{\mathcal{F}}$ is defined as in Proposition 4.4. Therefore, $0 \in \cap_{A \in \hat{\mathcal{F}}} W(A)$. Hence, by Proposition 4.4 and Theorem $3.5, W(\mathcal{F})=W(\hat{\mathcal{F}})$ is star-shaped.

Recall that $W_{C}(\mathcal{F})$ may fail to be convex even if $\mathcal{F}$ is convex and $W_{C}(A)$ is convex for all $A \in \mathcal{F}$. In the following, we give a necessary and sufficient condition for $W_{C}(\mathcal{F})$ to be convex. First, it is easy to see that $W_{C}(A) \subseteq W_{C}(\mathcal{F})$ for every $A \in \mathcal{F}$. Thus, $\operatorname{conv}\left\{\cup_{A \in \mathcal{F}} W_{C}(A)\right\}$ is the smallest convex set containing $W_{C}(\mathcal{F})$. As a result, we have the following observation.

Proposition 4.6. Let $C \in M_{n}$ and $\mathcal{F} \subseteq M_{n}$. Then $W_{C}(\mathcal{F})$ is convex if and only if

$$
W_{C}(\mathcal{F})=\operatorname{conv}\left\{\bigcup_{A \in \mathcal{F}} W_{C}(A)\right\} .
$$

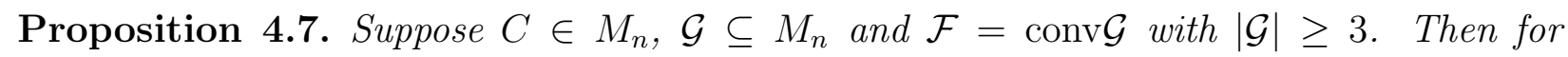
every $\mu \in W_{C}(\mathcal{F})$, there are matrices $A_{1}, A_{2}, A_{3} \in \mathcal{G}$ and a unitary matrix $U$ such that $\mu \in \operatorname{conv}\left\{\operatorname{tr}\left(C U^{*} A_{1} U\right), \operatorname{tr}\left(C U^{*} A_{2} U\right), \operatorname{tr}\left(C U^{*} A_{3} U\right)\right\}$. If $\mu$ is a boundary point of $W_{C}(\mathcal{F})$, then there are $B_{1}, B_{2} \in \mathcal{G}$ and a unitary $V$ such that $\mu \in \operatorname{conv}\left\{\operatorname{tr}\left(C V^{*} B_{1} V\right), \operatorname{tr}\left(C V^{*} B_{2} V\right)\right\}$.

Proof. For any $\mu \in W_{C}(\mathcal{F})$, we have $\mu=t_{1} \operatorname{tr}\left(C U^{*} A_{1} U\right)+\cdots+t_{r} \operatorname{tr}\left(C U^{*} A_{r} U\right)$ for some unitary matrix $U$ and $A_{1}, \ldots, A_{r} \in \mathcal{G}$ with $t_{1}, \ldots, t_{r}>0$ summing up to 1 . So, $\mu \in$ $\operatorname{conv}\left\{\operatorname{tr}\left(C U^{*} A_{1} U\right), \ldots, \operatorname{tr}\left(C U^{*} A_{r} U\right)\right\}$. Thus, $\mu$ lies in the convex hull of no more than three of the points in $\left\{\operatorname{tr}\left(C U^{*} A_{1} U\right), \ldots, \operatorname{tr}\left(C U^{*} A_{r} U\right)\right\}$. In case $\mu$ is a boundary point of $W_{C}(\mathcal{F})$, 
then $\mu$ must be a boundary point of the set $\operatorname{conv}\left\{\operatorname{tr}\left(C U^{*} A_{1} U\right), \ldots, \operatorname{tr}\left(C U^{*} A_{r} U\right)\right\}$. Thus, $\mu$ list in the convex hull of no more than two points in the set $\left\{\operatorname{tr}\left(C U^{*} A_{1} U\right), \ldots, \operatorname{tr}\left(C U^{*} A_{r} U\right)\right\}$. The assertion follows.

Theorem 4.8. Suppose $C \in M_{n}, \mathcal{G} \subseteq M_{n}$ is compact and $\mathcal{F}=\operatorname{conv}(\mathcal{G})$. The following are equivalent.

(a) $W_{C}(\mathcal{F})$ is convex.

(b) $W_{C}(\mathcal{F})$ is simply connected and every boundary point $\mu \in \operatorname{conv} W_{C}(\mathcal{F})$ has the form $\operatorname{tr}\left(C U^{*}(t A+(1-t) B) U\right)$ for some unitary matrix $U, t \in[0,1]$ and $A, B \in \mathcal{G}$.

Proof. Suppose $W_{C}(\mathcal{F})$ is convex. Then it is clearly simply connected. Now, conv $W_{C}(\mathcal{F})$ and $W_{C}(\mathcal{F})$ have the same boundary. By Proposition 4.7, every boundary point of $W_{C}(\mathcal{F})$ is a convex combination of $\operatorname{tr}\left(C U^{*} A U\right), \operatorname{tr}\left(C U^{*} B U\right)$ with $A, B \in \mathcal{G}$.

Conversely, suppose (b) holds. We only need to show that each boundary point $\mu$ of $\operatorname{conv}\left(W_{C}(\mathcal{F})\right)$ lies in $W_{C}(\mathcal{F})$, which is true by Proposition 4.7 and assumption (b).

\section{Extension to the joint $C$-numerical range}

Let $A=A_{1}+i A_{2} \in M_{n}$, where $A_{1}, A_{2} \in M_{n}$ are Hermitian matrices. Then $W(A)$ can be identified as the joint numerical range of $\left(A_{1}, A_{2}\right)$ defined by

$$
W\left(A_{1}, A_{2}\right)=\left\{\left(x^{*} A_{1} x, x^{*} A_{2} x\right): x \in \mathbb{C}^{n}, x^{*} x=1\right\}
$$

One may consider whether our results can be extended to the joint numerical range of an $m$-tuple of Hermitian matrices $\left(A_{1}, \ldots, A_{m}\right)$ defined by

$$
W\left(A_{1}, \ldots, A_{m}\right)=\left\{\left(x^{*} A_{1} x, \ldots, x^{*} A_{m} x\right): x \in \mathbb{C}^{n}, x^{*} x=1\right\} .
$$

Some of the results on classical numerical range are not valid for the joint numerical range. For instance, the joint numerical range of three matrices may not be convex if $n=2$. For $A_{1}=\left(\begin{array}{ll}0 & 1 \\ 1 & 0\end{array}\right), A_{2}=\left(\begin{array}{cc}0 & -i \\ i & 0\end{array}\right), A_{3}=\left(\begin{array}{cc}1 & 0 \\ 0 & -1\end{array}\right)$, we have

$$
W\left(A_{1}, A_{2}, A_{3}\right)=\left\{(a, b, c): a, b, c \in \mathbb{R}, a^{2}+b^{2}+c^{2}=1\right\} .
$$

The following is known, see [1, 12].

1. Suppose $A_{1}, A_{2}, A_{3} \in M_{2}$ are Hermitian matrices such that $\left\{I_{2}, A_{1}, A_{2}, A_{3}\right\}$ is linearly independent. Then $W\left(A_{1}, A_{2}, A_{3}\right)$ is an ellipsoid without interior in $\mathbb{R}^{3}$.

2. If $n \geq 3$ and $A_{1}, A_{2}, A_{3} \in M_{n}$ are Hermitian matrices, then $W\left(A_{1}, A_{2}, A_{3}\right)$ is convex. 
3. Suppose $A_{1}, A_{2}, A_{3} \in M_{n}$ such that $\left\{I_{2}, A_{1}, A_{2}, A_{3}\right\}$ is linearly independent. Then there is $A_{4} \in M_{n}$ such that $W\left(A_{1}, A_{2}, A_{3}, A_{4}\right)$ is not convex.

There has been study of topological and geometrical properties of $W\left(A_{1}, \ldots, A_{m}\right)$. Researchers also consider the joint $C$-numerical range of $\left(A_{1}, \ldots, A_{m}\right)$ defined by

$$
W_{C}\left(A_{1}, \ldots, A_{m}\right)=\left\{\left(\operatorname{tr}\left(C U^{*} A_{1} U\right), \ldots, \operatorname{tr}\left(C U^{*} A_{m} U\right)\right): U \text { unitary }\right\}
$$

for a Hermitian matrix $C$, for example [5, 6]. In particular, it is known that $W_{C}\left(A_{1}, A_{2}, A_{3}\right)$ is convex for any Hermitian matrices $C, A_{1}, A_{2}, A_{3} \in M_{n}$ if $n \geq 3$, see [2]. Of course, one can also consider the $C$-numerical range of $\left(A_{1}, \ldots, A_{m}\right)$ for general matrices $C, A_{1}, \ldots, A_{m} \in M_{n}$ defined as in Equation (5.3).

Denote by $\mathbf{A}=\left(A_{1}, \ldots, A_{m}\right)$ an $m$-tuple of matrices in $M_{n}$. Let $\mathbf{F}$ be a non-empty subset of $M_{n}^{m}$. We consider

$$
W_{C}(\mathbf{F})=\bigcup\left\{W_{C}(\mathbf{A}): \mathbf{A} \in \mathbf{F}\right\} .
$$

Evidently, when $\mathbf{F}=\{\mathbf{A}\}$, then $W_{C}(\mathbf{F})=W_{C}(\mathbf{A})$.

\subsection{Basic results}

We begin with the following results.

Proposition 5.1. Let $C \in M_{n}$ be non-scalar, and let $\mathbf{F}$ be a non-empty subset of $M_{n}^{m}$.

1. For any unitary $U, V \in M_{n}$, we have $W_{C}(\mathbf{F})=W_{V^{*} C V}\left(U^{*} \mathbf{F} U\right)$, where

$$
U^{*} \mathbf{F} U=\left\{\left(U^{*} A_{1} U, \ldots, U^{*} A_{m} U\right):\left(A_{1}, \ldots, A_{m}\right) \in \mathbf{F}\right\}
$$

2. Let $\gamma_{1}, \gamma_{2} \in \mathbb{C}$ with $\gamma_{1} \neq 0$. If $\hat{C}=\gamma_{1} C+\gamma_{2} I_{n}$, then for any $\mathbf{A}=\left(A_{1}, \ldots, A_{m}\right) \in M_{n}^{m}$,

$$
W_{\hat{C}}(\mathbf{A})=\left\{\gamma_{1}\left(a_{1}, \ldots, a_{m}\right)+\gamma_{2}\left(\operatorname{tr} A_{1}, \ldots, \operatorname{tr} A_{m}\right):\left(a_{1}, \ldots, a_{m}\right) \in W_{C}(\mathbf{A})\right\} .
$$

3. For any $T=\left(t_{i j}\right) \in M_{m}$ and $f=\left(f_{1}, \ldots, f_{m}\right)^{t}$, we can define an affine map $R$ on $\mathbb{C}^{m}$ by $v \mapsto T v+f$, and extend the affine map to $M_{n}^{m}$ by mapping $\mathbf{A}=\left(A_{1}, \ldots, A_{m}\right)$ to $\mathbf{B}=\left(B_{1}, \ldots, B_{m}\right)$ with $B_{i}=\sum_{j=1}^{n} t_{i j} A_{j}+f_{i} I_{n}$. Then

$$
\begin{aligned}
& W_{C}(\mathbf{B})=\left\{\left(b_{1}, \ldots, b_{m}\right)\right.:\left(b_{1}, \ldots, b_{m}\right)^{t}=T\left(a_{1}, \ldots, a_{n}\right)^{t}+ \\
&\left.(\operatorname{tr} C)\left(f_{1}, \ldots, f_{m}\right)^{t},\left(a_{1}, \ldots, a_{m}\right) \in W_{C}(\mathbf{A})\right\} .
\end{aligned}
$$

Consequently,

$$
R\left(W_{C}(\mathbf{F})\right)=W_{C}(R(\mathbf{F})) .
$$

4. The linear span of $\left\{A_{j}-\left(\operatorname{tr} A_{j}\right) / n: j=1, \ldots, m\right\}$ has dimension $k$ if and only if $W_{C}(\mathbf{F}) \subseteq \mathbf{V}+f$ for a $k$-dimensional subspace $\mathbf{V} \subseteq \mathbb{C}^{m}$ and a vector $f \in \mathbb{C}^{m}$. In particular, $W_{C}(\mathbf{F})$ is a singleton $\left\{\left(\nu_{1}, \ldots, \nu_{n}\right)\right\}$ if and only if $\mathcal{F}=\left\{\left(\mu_{1} I, \ldots, \mu_{m} I\right)\right\}$ with $(\operatorname{tr} C)\left(\mu_{1}, \ldots, \mu_{m}\right)=\left(\nu_{1}, \ldots, \nu_{n}\right)$. 
Note that if $C \in M_{n}$ is Hermitian, then $W_{C}\left(A_{1}, \ldots, A_{m}\right) \subseteq \mathbb{C}^{m}$ can be identified as $W_{C}\left(X_{1}, Y_{1}, X_{2}, Y_{2}, \ldots, X_{m}, Y_{m}\right) \subseteq \mathbb{R}^{2 m}$, where $X_{j}=\left(A_{j}+A_{j}^{*}\right) / 2$ and $Y_{j}=\left(A_{j}-A_{j}^{*}\right) /(2 i)$. One can obtain a "real version" of Proposition 5.1 using real scalars $\gamma_{1}, \gamma_{2}$, real matrix $T$, real vector $f$, etc.

One can prove the following when $W_{C}(\mathbf{F})$ is a polyhedral set in $\mathbb{C}^{m}$, i.e., a convex combination of a finite set of vertices.

Proposition 5.2. Suppose $C \in M_{n}$ is non-scalar, and $\mathbf{F}$ is a non-empty set of $M_{n}^{m}$. If $W_{C}(\mathbf{F})$ is polyhedral, then every vertex has the form $\left(\mu_{1}, \ldots, \mu_{m}\right)$ with $\mu_{j}=\operatorname{tr} V^{*} C V U^{*} A_{j} U$, where $\left(A_{1}, \ldots, A_{m}\right) \in \mathbf{F}, U, V \in M_{n}$ are unitary so that $V^{*} C V, U^{*} A_{1} U, \ldots, U^{*} A_{m} U$ are in lower triangular matrices with diagonal entries

$$
c_{1}, \ldots, c_{n}, a_{1}(1), \ldots, a_{n}(1), \ldots, a_{1}(m), \ldots, a_{n}(m)
$$

and $\mu_{j}=\sum_{\ell=1}^{n} c_{\ell} a_{\ell}(j)$. Furthermore, if $c_{1}, \ldots, c_{n}$ are distinct, then

$$
V^{*} C V, U^{*} A_{1} U, \ldots, U^{*} A_{m} U
$$

are diagonal matrices.

If $C$ has distinct eigenvalues, and if $W_{C}(\mathbf{A})$ has a conical point $\left(\mu_{1}, \ldots, \mu_{m}\right)$ on the boundary, i.e., there is a pointed cone $K \subseteq \mathbb{C}^{m} \equiv \mathbb{R}^{2 m}$ with vertex $\left(\mu_{1}, \ldots, \mu_{m}\right)$ such that

$$
W_{C}(\mathbf{A}) \cap\left\{\left(\mu_{1}+\nu_{1}, \ldots, \mu_{m}+\nu_{m}\right):\left|\nu_{j}\right| \leq \varepsilon\right\} \subseteq K
$$

for some sufficiently small $\varepsilon>0$, then $\left\{C, A_{1}, \ldots, A_{m}\right\}$ is a commuting family of normal matrices, and conv $W_{C}(\mathbf{A})$ is a polyhedral set, see [3].

Note that if there is $\mathbf{B} \in \mathbf{F}$ such that $W_{C}(\mathbf{B})$ is a singleton, a subset of a straight line, or a convex polygon, then we can apply the results on $W_{C}(\mathbf{B})$ to deduce that $C$ and $\mathbf{B}$ has special structure. Then we can deduce results on $W_{C}(\mathbf{F})$.

We can obtain some topological properties $W_{C}(\mathbf{F})$.

Proposition 5.3. Let $C \in M_{n}$ be non-scalar, and $\mathbf{F} \subseteq M_{n}^{m}$ be a nonempty set.

1. If $\mathcal{F}$ is bounded, then so is $W_{C}(\mathbf{F})$.

2. If $\mathcal{F}$ is connected, then so is $W_{C}(\mathbf{F})$.

3. If $\mathcal{F}$ is compact, then so is $W_{C}(\mathbf{F})$.

Proof. The proof of the boundedness and compactness are similar to those for $W_{C}(\mathcal{F})$ in Section 2. The prove connectedness, for any $\mathbf{A}, \mathbf{B} \in \mathbf{F}$ and unitary $U_{0}, U_{1} \in M_{n}$, there is a path joining $U_{t}$ with $t \in[0,1]$ joining $U_{0}$ and $U_{t}$, and hence there is a path joining $\left(\operatorname{tr} U_{0}^{*} C U_{0} A_{1}, \ldots, \operatorname{tr} U_{0}^{*} C U_{0} A_{m}\right)$ to $\left(\operatorname{tr} U_{1}^{*} C U_{1} A_{1}, \ldots, \operatorname{tr} U_{1}^{*} C U_{1} A_{m}\right)$, which is connected to $\left(\operatorname{tr} U_{1}^{*} C U_{1} B_{1}, \ldots, \operatorname{tr} U_{1}^{*} C U_{1} B_{m}\right)$. 


\subsection{Star-shapedness and convexity}

Here we consider whether $W(\mathbf{F})$ is star-shaped or convex. It is known that if $C \in M_{n}$ is Hermitian, then $W_{C}\left(A_{1}, A_{2}, A_{3}\right)$ is convex for any Hermitian $A_{1}, A_{2}, A_{3} \in M_{n}$ with $n \geq 3$, see [2]. One may wonder whether Theorem 3.5 admits an extension to this setting. The following example shows that the answer is negative.

Example 5.4. Let

$$
\mathbf{A}_{t}=\left(\left(\begin{array}{lll}
t & & \\
& t & \\
& & 1-t
\end{array}\right),\left(\begin{array}{lll}
1-t & & \\
& t & \\
& & 1-t
\end{array}\right)\left(\begin{array}{lll}
0 & & \\
& t & \\
& & 1-t
\end{array}\right)\right), \quad t \in[0,1] .
$$

Notice that $\operatorname{conv}\left\{\mathbf{A}_{0}, \mathbf{A}_{1}\right\}=\left\{\mathbf{A}_{t}: 0 \leq t \leq 1\right\}$. Moreover, $(0,0,0) \in W\left(\mathbf{A}_{0}\right) \cap W\left(\mathbf{A}_{1}\right)$, but $(0,0,0)$ is not a star-center of $W\left(\operatorname{conv}\left\{\mathbf{A}_{0}, \mathbf{A}_{1}\right\}\right)$ as $(1 / 2,1 / 2,0) \in W\left(\mathbf{A}_{1 / 2}\right)$, however, $\operatorname{conv}\{(0,0,0),(1 / 2,1 / 2,0)\} \nsubseteq W\left(\operatorname{conv}\left\{\mathbf{A}_{0}, \mathbf{A}_{1}\right\}\right)$. Nevertheless, $(1 / 2,1 / 2,1 / 2) \in W\left(\mathbf{A}_{t}\right)$ for all $t \in[0,1]$, and hence $(1 / 2,1 / 2,1 / 2)$ is a star-center of $W\left(\operatorname{conv}\left\{\mathbf{A}_{0}, \mathbf{A}_{1}\right\}\right)$. Actually, one can show that $W\left(\mathbf{A}_{t}\right)=\operatorname{conv}\{(t, 1-t, 0),(t, t, t),(1-t, 1-t, 1-t)\}$.

Here is an example showing that $W(\mathbf{A})$ may not be star-shaped in general.

Example 5.5. Let $\mathbf{A}=\left(A_{1}, A_{2}, A_{3}\right)$ with $A_{1}=\operatorname{diag}(0,1,0), A_{2}=\operatorname{diag}(1,0,-1), A_{3}=I_{3}$ and let $\mathbf{B}=\left(B_{1}, B_{2}, B_{3}\right)$ with $B_{1}=\operatorname{diag}(1,0,0), B_{2}=\operatorname{diag}(0,-1,1), B_{3}=0_{3}$. If $\mathbf{F}=$ $\operatorname{conv}\{\mathbf{A}, \mathbf{B}\}$, then $W(\mathbf{F})$ is the union of the triangular disk with vertices

$$
(1-t, t, t),(t, t-1, t),(0,1-2 t, t) .
$$

Let $g: \mathbb{R}^{3} \rightarrow \mathbb{R}^{3}$ be given by $g((a, b, c))=(a,-b, 1-c)$. We have

$$
\begin{aligned}
g((1-t, t, t)) & =(1-t,-t, 1-t)=(1-t,(1-t)-1,1-t) \\
g((t, t-1, t)) & =(t, 1-t, 1-t)=(1-(1-t),(1-t),(1-t)) \\
g((0,1-2 t, t)) & =(0,2 t-1,1-t)=(0,1-2(1-t),(1-t))
\end{aligned}
$$

Therefore, $g(W(\mathbf{F}))=W(\mathbf{F})$. Moreover, we claim that $W(\mathbf{F})$ is not star-shaped.

Suppose the contrary that $W(\mathbf{F})$ is star-shaped with $\left(a, b, t_{0}\right)$ be a star-center. Then $\left(a,-b, 1-t_{0}\right)$ is also a star-center. As the set of all star-center of a star-shaped set is convex. We may now assume without loss of generality that $b=0$ and $t_{0}=1 / 2$. Assume now $a>0$. By assumption, for all $0 \leq t \leq 1, t(a, 0,1 / 2)+(1-t)(1,0,0)=(1-t(1-a), 0, t / 2) \in W(\mathbf{F})$. By direct computation, given $0 \leq t \leq 1$, we have

$$
\max \{\alpha \in \mathbb{R}:(\alpha, 0, t) \in W(\mathbf{F})\}=t^{2}+(1-t)^{2}=1-2 t+2 t^{2} .
$$

However for sufficient small $t>0$

$$
1-t(1-a)>1-t\left(1-t^{2} / 2\right)>(t / 2)^{2}+(1-t / 2)^{2}
$$


which contradicts that $(1-t(1-a), 0, t / 2) \in W(\mathbf{F})$. Therefore, we have $a=0$. However $(0,0,1 / 2)$ is not a star-center as

$$
\frac{1}{3}(0,0,1 / 2)+\frac{2}{3}(0,0,1)=\left(0,0, \frac{5}{6}\right) \notin \operatorname{conv}\left\{\left(\frac{1}{6}, \frac{5}{6}, \frac{5}{6}\right),\left(\frac{5}{6},-\frac{1}{6}, \frac{5}{6}\right),\left(0,-\frac{2}{3}, \frac{5}{6}\right)\right\}
$$

Therefore, $W(\mathbf{F})$ is not star-shaped.

It is challenging to determine conditions on $\mathbf{F}$ so that $W(\mathbf{F})$ is star-shaped.

\section{Acknowledgment}

Our study was inspired by some discussion at the AIM workshop on Crouzeix's conjecture, July 30 - August 5, 2017, AIM, San Jose. The second and the third authors would like to express their thanks to the organizer and the colleagues at AIM for the well-organized and stimulating workshop.

$\mathrm{Li}$ is an honorary professor of the Shanghai University, and an affiliate member of the Institute for Quantum Computing, University of Waterloo; his research was supported by the USA NSF DMS 1331021, the Simons Foundation Grant 351047, and NNSF of China Grant 11571220. Research of Sze and Lau were supported by a PolyU central research grant G-YBKR and a HK RGC grant PolyU 502512. The HK RGC grant also supported the post-doctoral fellowship of Lau at the Hong Kong Polytechnic University.

\section{References}

[1] Y. Au Yeung and Y.T. Poon, A remark on the convexity and positive definiteness concerning Hermitian matrices, Southeast Bull. Math. 3 (1979), 85-92.

[2] Y. Au Yeung and N.K. Tsing, An extension of the Hausdorff-Toeplitz theorem on the numerical range, Proc. Amer. Math. Soc. 89 (1983), 215-218.

[3] P. Binding and C. K. Li, Joint ranges of hermitian matrices and simultaneous diagonalization, Linear Algebra Appl. 151 (1991), 157-168.

[4] W.S. Cheung and N.K. Tsing, The $C$-numerical range of matrices is star-shaped, Linear and Multilinear Algebra 41 (1996), 245-250.

[5] M.T. Chien and H. Nakazato, Strict convexity of the joint c-numerical range, Linear Algebra Appl. 438 (2013), 1305-1321.

[6] M.D. Choi, C.K. Li and Y.T. Poon, Some convexity features associated with unitary orbits, Canad. J. Math. 55 (2003), 91-111.

[7] M. Crouzeix, Bounds for analytical functions of matrices, Integral Equations Operator Theory 48 (2004), 461477. 
[8] P.R. Halmos, A Hilbert Space Problem Book, Spring-Verlag, New York, 1982.

[9] R.A. Horn and C.R. Johnson, Topics in Matrix Analysis, Cambridge University Press, Cambridge,1991.

[10] C.K. Li, $C$-numerical ranges and $C$-numerical radii, Linear and Multilinear Algebra 37 (1994), 51-82.

[11] C.K. Li, D.C. Pelejo, Y.T. Poon and K.Z. Wang, Minkowski product of convex sets and product numerical range, Operators and Matrices, 10 (2016), 945-965.

[12] C.K. Li and Y.T. Poon, Convexity of the joint numerical range, SIAM J. Matrix. Anal. Appl. 21 (2000), 668-678.

[13] C.K. Li and N.K. Tsing, Matrices with circular symmetry on their unitary orbits and $C$-numerical ranges, Proc. of Amer. Math. Soc. 111 (1991), 19-28.

[14] H. Nakazato, The $C$-numerical range of a $3 \times 3$ normal matrix, Nihonkai Math. J. 17 (2006), 187-197.

[15] Z. Puchala, P. Gawron, J.A. Miszczak, L. Skowronek, M.D. Choi, and K. Zyczkowski, Product numerical range in a space with tensor product structure, Linear Algebra Appl. 434 (2011), 327-342.

[16] T. Schulte-Herbrüggen , G. Dirr , U.Helmke and S.J. Glaser The significance of the $C$-numerical range and the local $C$-numerical range in quantum control and quantum information, Linear and Multilinear Algebra 56 (2008), 3-26.

[17] N.K. Tsing, The constraint bilinear form and the $C$-numerical range, Linear Algebra Appl. 56 (1984), 195-162.

[18] R. Westwick, A theorem on numerical range, Linear and Multilinear Algebra 2 (1975), 311-315.

\section{Addresses}

(P.S. Lau) Department of Applied Mathematics, The Hong Kong Polytechnic University, Hung Hom, Hong Kong. (panshun.lau@polyu.edu.hk)

(C.K. Li) Department of Mathematics, College of William \& Mary, Williamsburg, VA 23185. (ckli@math.wm.edu)

(Y.T. Poon) Department of Mathematics, Iowa State University, Ames, IA 50011.

(ytpoon@iastate.edu)

(N.S. Sze) Department of Applied Mathematics, The Hong Kong Polytechnic University, Hung Hom, Hong Kong. (raymond.sze@polyu.edu.hk) 